\title{
Realizations of Voltage Transfer Functions Using DVCCs
}

\section{Tejmal S. Rathore}

School of Electrical Sciences, IIT Goa, Ponda, India

Email: tsrathor@ee.iitb.ac.in, tsrathore@iitgoa.ac.in

How to cite this paper: Rathore, T.S. (2018) Realizations of Voltage Transfer Functions Using DVCCs. Circuits and Systems, 9, 141-147.

https://doi.org/10.4236/cs.2018.910015

Received: July 30, 2018

Accepted: October 27, 2018

Published: October 31, 2018

Copyright (C) 2018 by author and Scientific Research Publishing Inc. This work is licensed under the Creative Commons Attribution International License (CC BY 4.0).

http://creativecommons.org/licenses/by/4.0/

\begin{abstract}
Maheshwari has proposed three differential-voltage current-conveyor configurations for realizing first order all-pass filters only. This paper has exploited these configurations for realizing more complex transfer function $T(s)$ which yield poles and zeros of $1-T(s)$ in one of the four admissible patterns. Bilinear and biquadratic functions are dealt in detail. It is shown that only bilinear functions can be realized with all the four passive elements grounded. First order all-pass function is a special case which needs only three elements $(2 R, 1 C)$ or $(1 R, 2 C)$. A biquadratic function requires $(2 R, 2 C)$ elements and has all the capacitor grounded. Design of second order all-pass function is given.
\end{abstract}

\section{Keywords}

Differential-Voltage Current-Conveyor, All-Pass Filters, Bilinear Functions, Biquadratic Functions

\section{Introduction}

The symbol of a difference voltage current conveyor (DVCC) is shown in Figure 1 and its characteristics are summarized by the following port relationships [1]

$$
V_{X}=V_{Y 1}-V_{Y 2}, I_{Y 1}=I_{Y 2}=0, I_{Z+}=I_{X}, I_{Z-}=-I_{X}
$$

Maheshwari [2] has proposed three configurations, shown in Figure 2, using DVCC for realizing only first order voltage-mode all-pass filter. The filter has the advantages of high input resistance and minimum number of RC elements and all of them grounded.

The intent of this paper is to exploit the circuit topologies of Figure 2 to realize more general class of functions. Conditions of realization are derived. Then we consider realizations of bilinear and biquadratic functions in detail. In the 


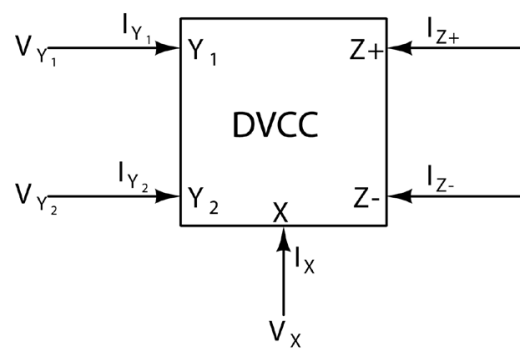

Figure 1. Block representation of a differential voltage current conveyor.

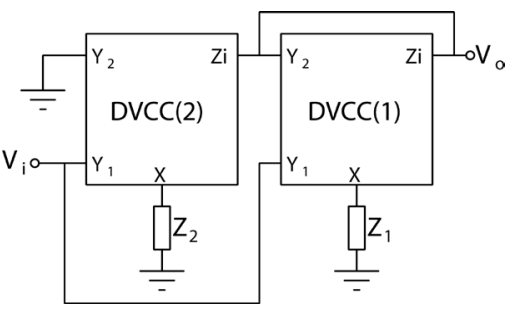

Figure 2. Proposed topologies where zi's in DVCC (1) and (2) are (a) z+, z-; (b) $\mathrm{z}_{-}^{-}, \mathrm{z}+$, (c) $\mathrm{z}+, \mathrm{z}+$, respectively.

former case, it is shown that only first order functions can have all passive components grounded, and that there are only two possible cases with minimum number of passive elements ( $1 C 2 R$ and $2 C 1 R$ ) logically, rather than intuitionally in [2]. In case of biquadratic function only 4 passive elements $(2 C, 2 R)$ are required with both the capacitors grounded. Desing of a second order all-pass function is given.

\section{Realization of a General Voltage Transfer Function}

Each of the circuit topologies of Figures 2(a)-(c) has the voltage transfer function

$$
T(s)=K \frac{N(s)}{D(s)}=1-Z_{1} Y_{2}
$$

From (2),

$$
Z_{1} Y_{2}=\frac{D(s)-K N(s)}{D(s)}
$$

Note that the poles of $Z_{1} Y_{2}$ are the same as those of $T$; but zeros are given by $D$ - $K N=0$. Impedances $Z_{1}$ and $Z_{2}$ can be identified as $R C$ driving point functions (DPIs) from (3), if the poles and zeros of $Z_{1} Y_{2}$, arranged in pairs starting from the rightmost pair, each pair consists of a pole and a zero in either order [3]. The four admissible pole-zero patterns are shown in Figure 3. $T(\mathrm{~s})$, so also $Z_{1} Y_{2}$, must have poles distinct negative real. Zero lociof $Z_{1} Y_{2}$ start from the poles when $K=0$ and terminate on the zeros of $T(s)$ when $K=\infty$. Hence it is possible to choose $K$ sufficiently small so that the zeros are negative real. Thus, if the poles and zeros of $Z_{1} Y_{2}$ fit into one of the patterns shown in Figure 3, $T$ is realizable otherwise not. 


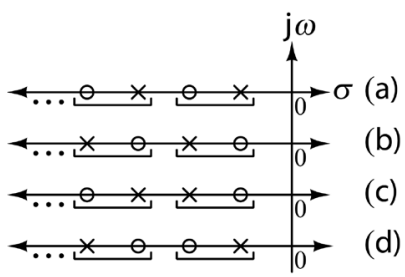

Figure 3. Admissible pole zero patterns.

\subsection{Realization of Bilinear Voltage Transfer Functions}

Let the bilinear voltage transfer function be

$$
T(s)=\frac{K(s+z)}{s+p}=1-Z_{1} Y_{2}
$$

If $z$ is positive, i.e., it lies on the negative real axis, then $T(s)$ can be realized by RC passive elements. Therefore, we shall consider the case when $z$ is negative i.e., $0 \leq z \leq \infty$.

Then

$$
Z_{1} Y_{2}=\frac{(1-K) s+(p+K z)}{s+p}
$$

The zero-locus with $K$ as variable is shown in Figure 4. It starts from pole at $-p$ when $K=0$ and reaches $-\infty$ when $K=1$ and again from $+\infty$ to zero at $z$ from $K=1$ to $K=\infty$. Thus choosing $0<K \leq 1$, the zero of $Z_{1} Y_{2}$ can be made negative real. Thus it will follow the pattern shown in Figure $3(a)$ and Figure $3(d)$. Now

$$
Z_{1} Y_{2}=\mu \frac{s+\alpha}{s+p}, \quad p<\alpha \leq \infty .
$$

Only possible identifications are

$$
\begin{gathered}
Z_{1}=\frac{\mu_{1}}{s+p}, \quad Y_{2}=\mu_{2}(s+\alpha) \\
Z_{1}=\mu_{1} \frac{s+\alpha}{s}, \quad Y_{2}=\mu_{2} \frac{s}{s+p} \\
Z_{1}=\mu_{1} \frac{s+\alpha}{s+p}, \quad Y_{2}=\mu_{2}
\end{gathered}
$$

where $\mu=\mu_{1} \mu_{2}$. The possible canonic realizations of $Z_{1}$ and $Z_{2}$ in Foster and Cauer forms from (7) is given in Figure 5(a), from (8) in Figure 5(b), and from (9) in Figure 5(c) and Figure 5(d), respectively.

Minimum 4 elements $(2 C, 2 R)$ or $(1 C, 3 R)$ are required for realizing $Z_{1,2}$ as shown in Figure 5 . However, one element can be reduced by choosing $K=1$ which forces the zero of $Z_{1} Y_{2}$ at $\infty$ (see (5)). The reduced realizations of $Z_{1,2}$ are shown in Figure 6. The complete realizations of $T(s)$ given by (4) is obtained by inserting $Z_{1,2}$ of Figure 6 in Figure 2. They reduce to all-pass functions when $p=$ $z$. Thus we get $(2 R, 1 C)$ and $(1 R, 2 C)$ realizations for first order all-pass function. The realization corresponding to $(2 R, 1 C)$ has all the passive elements grounded. We have thus shown that there are four possible realizations and only one of 


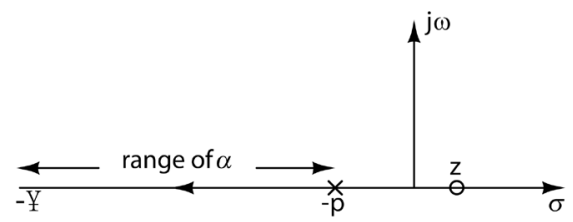

Figure 4. Root locus with $K$ as variable.

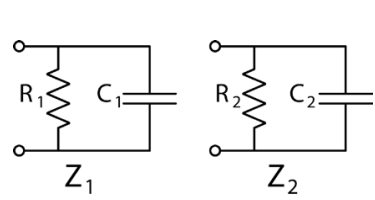

(a)

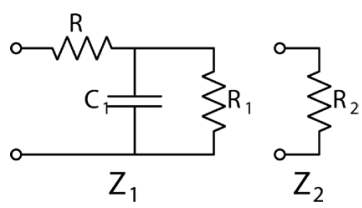

(c)

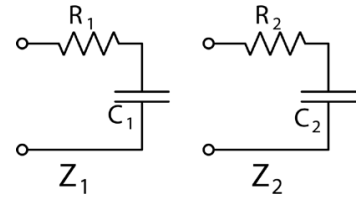

(b)

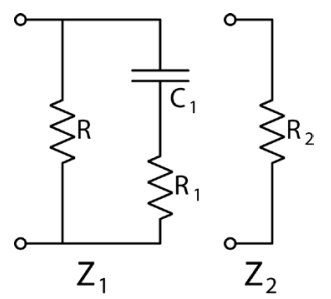

(d)

Figure 5. Realizations of $Z_{1}$ and $Z_{2}$ given by (a) (7); (b) (8) and (c) and (d) by (9).

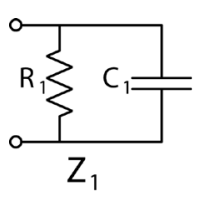

(a)

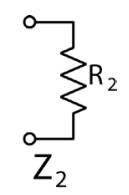

)
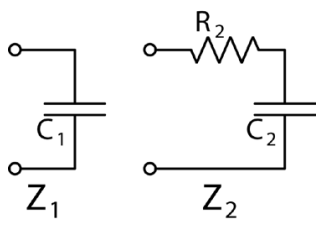

(b)

Figure 6. Two realizations of $Z_{1,2}$.

which have all three elements $(2 R, 1 C)$ grounded systematically, and not intuitively as in [2].

\section{Alternative proof}

In Figure 2, $Z_{1}$ and $Z_{2}$ can have only one $R$ and one $C$ element at the most in parallel for them to be grounded. Any additional resistance (capacitance) in parallel will be absorbed in $R(C)$ already present. Let $Z_{i}(i=1,2)$ be parallel combinations of one $R_{i}$ and one $C_{i}$ as shown in the Figure 5(d). Then

$$
Z_{i}=\left(\frac{1}{C_{i}}\right) \frac{1}{\left(s+\frac{1}{C_{i} R_{i}}\right)}, \quad i=1,2
$$

Now

$$
T(s)=1-\frac{Z_{1}}{Z_{2}}=\left(1-\frac{C_{2}}{C_{1}}\right) \frac{s-\frac{1}{\left(1-\frac{C_{2}}{C_{1}}\right)}\left(\frac{R_{1}}{R_{2}}-1\right) \frac{1}{C_{1} R_{1}}}{\left\{s+\frac{1}{C_{1} R_{1}}\right\}}=\mu \frac{\{s-\alpha\}}{\{s+p\}}
$$


Since the denominator is of first order, the circuit can realize only bilinear transfer functions with four components $(2 C$ and $2 R$ ); all grounded. The number of elements can be reduced by 1 under specific conditions. Let us consider the special case of all pass function. From (11), the condition is

$$
Z_{i}=\left(\frac{1}{C_{i}}\right) \frac{1}{\left(s+\frac{1}{C_{i} R_{i}}\right)}, \quad i=1,2
$$

There are infinite number of solutions to satisfy (11). From (11), it is obvious that $R_{1}$ cannot be equal to $R_{2}$ and $C_{1}$ cannot be equal to $C_{2}$. To have minimum number of passive components, the choices are $C_{2}=0, R_{1}=2 R_{2}$ and $R_{2}=\infty, C_{2}=$ $2 C_{1}$. In the latter choice, it can be seen from (11) that $T(s)$ will be negative. These choices were directly chosen in [2] without any logic.

\subsection{Realization of Biquadratic Transfer Functions}

Let the function be expressed as

$$
T(s)=K \frac{\left(s+z_{1}\right)\left(s+z_{2}\right)}{\left(s+p_{1}\right)\left(s+p_{2}\right)}
$$

where poles $p_{1,2}$, as discussed above, have to be negative real and $z_{1,2}$ may lie anywhere in the $s$-plane. Then from (2)

$$
Z_{1} Y_{2}=\frac{(1-K) s^{2}+\left[\left(p_{1}+p_{2}\right)-K\left(z_{1}+z_{2}\right)\right] s+\left(p_{1} p_{2}-K z_{1} z_{2}\right)}{\left(s+p_{1}\right)\left(s+p_{2}\right)}
$$

To realize with minimum number of elements, we choose $K=1$. Then

$$
Z_{1} Y_{2}=\frac{\left[\left(p_{1}+p_{2}\right)-\left(z_{1}+z_{2}\right)\right] s+\left(p_{1} p_{2}-z_{1} z_{2}\right)}{\left(s+p_{1}\right)\left(s+p_{2}\right)}=\mu \frac{(s+\alpha)}{\left(s+p_{1}\right)\left(s+p_{2}\right)}
$$

where $\mu=\left(p_{1}+p_{2}\right)-\left(z_{1}+z_{2}\right)$ and $\alpha=\frac{p_{1} p_{2}-z_{1} z_{2}}{\left(p_{1}+p_{2}\right)-\left(z_{1}+z_{2}\right)}$. If $\alpha$ and $p_{1,2}$ satisfy any of the pole-zero patterns shown in Figure 3 then $Z_{1,2}$ can be identified as driving point impedances. Since there are many possible locations of $Z_{1,2}$, we explain the procedure by taking the all-pass function for which $Z_{1,2}=-p_{1,2}$. Then (15) reduces to

$$
Z_{1} Y_{2}=\mu \frac{s}{\left(s+p_{1}\right)\left(s+p_{2}\right)}
$$

where $\mu=2\left(p_{1}+p_{2}\right)$.

Now $Z_{1,2}$ can be identified, in two possible ways as

$$
Z_{1}=\frac{\mu_{1}}{s+p_{1,2}}, \quad Z_{2}=\frac{s+p_{2,1}}{\mu_{2} s}
$$

where $\mu=\mu_{1} \mu_{2}$.

Choosing $\mu_{1}=\mu_{2}=1$, Two realizations of $Z_{1,2}$ given by (17) are shown in Figure 7. As expected, all elements are not grounded. It is interesting to realize an all-pass filter with double poles, i.e., $p_{1}=p_{2}=p$. In this case (17) reduces to 


$$
Z_{1}=\frac{\mu_{1}}{s+p}, \quad Z_{2}=\frac{s+p}{\mu_{2} s}
$$

Example: Realize a second order all-pass function

$$
T(s)=\frac{s^{2}-4 s+3}{s^{2}+4 s+3} .
$$

Here

$$
Z_{1} Y_{2}=1-T(s)=\frac{8 s}{(s+1)(s+3)}
$$

Identifying

$$
Z_{1}=\frac{1}{s+1}, \quad Z_{2}=\frac{s+3}{8 s}
$$

the complete realization of $T(s)$ of (19) is given in Figure 8.

\section{Conclusion}

Maheshwari [1] proposed three DVCC configurations and used them for realizing only first order all-pass filters. This paper has exploited these configurations for realizing transfer function $T(s)$ such that the location of poles and zeros of 1 $T(s)$ matches with any one shown in Figure 3 . It has been proved that only bilinear functions can be realized with all the four passive elements grounded. First order all-pass function is a special case which needs only three elements $(2 R, 1 C)$ or $(1 R, 2 C)$. Biquadratic functions have also been considered. They require $(2 C$, $2 R$ ) passive elements with both the capacitors grounded. We have not attempted here the non-ideal analysis, simulation and applications as they are very well documented in [1].

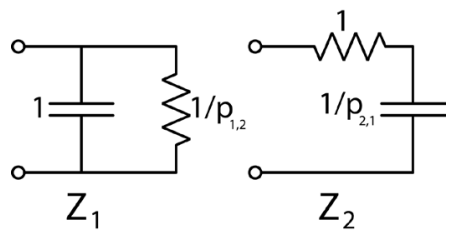

Figure 7. Two realizations of $Z_{1,2}$ given by (17).

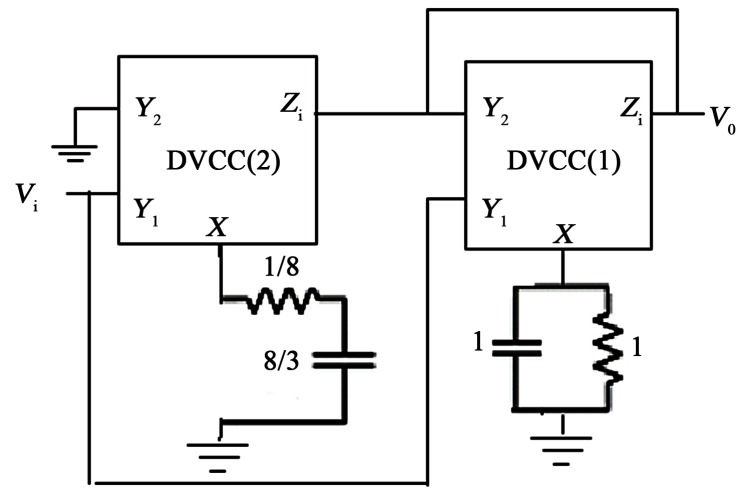

Figure 8. Realization of all pass function given by (19). 


\section{Conflicts of Interest}

The author declares no conflicts of interest regarding the publication of this paper.

\section{References}

[1] Ibrahim, M.A., Minaei, S. and Kuntman, H. (2005) A $22.5 \mathrm{MHz}$ Biquad Using Differential Voltage Current Conveyor and Grounded Passive Elements. International Journal of Electronics and Communications, 59, 311-318.

[2] Maheshwari, S. (2007) High Input Impedance VM-APSs with Grounded Passive Elements. IET Circuits, Devices and Systems, 1, 72-78.

https://doi.org/10.1049/iet-cds:20060196

[3] Rathore, T.S. and Khot, U.P. (2005) Single FTFN Realization of Current Transfer Functions. IETE Journal of Research, 51, 193-199.

https://doi.org/10.1080/03772063.2005.11416395 